\title{
openheart Performance of a clinical/proteomic panel to predict obstructive peripheral artery disease in patients with and without diabetes mellitus
}

Cian P McCarthy, ${ }^{1}$ Shreya Shrestha, ${ }^{1}$ Nasrien Ibrahim, ${ }^{2}$ Roland R J van Kimmenade, ${ }^{3}$ Hanna K Gaggin, ${ }^{4}$ Renata Mukai, ${ }^{1}$ Craig Magaret, ${ }^{5}$ Grady Barnes, ${ }^{5}$ Rhonda Rhyne, ${ }^{5}$ Joseph M Garasic, ${ }^{4}$ James L Januzzi ${ }^{4}$

- Additional material is published online only. To view please visit the journal online (http://dx.doi.org/10.1136/ openhrt-2018-000955).

To cite: McCarthy $\mathrm{CP}$, Shrestha S, Ibrahim N, et al. Performance of a clinical/ proteomic panel to predict obstructive peripheral artery disease in patients with and without diabetes mellitus. Open Heart 2019;6:e000955. doi:10.1136/ openhrt-2018-000955

Received 20 0ctober 2018 Revised 21 March 2019 Accepted 14 April 2019

D Check for updates

(C) Author(s) (or their employer(s)) 2019. Re-use permitted under CC BY-NC. No commercial re-use. See rights and permissions. Published by BMJ.

${ }^{1}$ Medicine, Massachusetts General Hospital, Boston, Massachusetts, USA

${ }^{2}$ Medicine/Cardiology, Massachusetts General Hospital Boston, Massachusetts, USA ${ }^{3}$ Cardiology, AZM, Maastricht, The Netherlands

${ }^{4}$ Cardiology, Massachusetts General Hospital, Boston,

Massachusetts, USA

${ }^{5}$ Prevencio, Kirkland,

Washington, USA

Correspondence to

James L Januzzi; JJANUZZI@

PARTNERS.ORG

\section{ABSTRACT}

Background Patients with diabetes mellitus (DM) are at substantial risk of developing peripheral artery disease (PAD). We recently developed a clinical/proteomic panel to predict obstructive PAD. In this study, we compare the accuracy of this panel for the diagnosis of PAD in patients with and without DM.

Methods and results The HART PAD panel consists of one clinical variable (history of hypertension) and concentrations of six biomarkers (midkine, kidney injury molecule-1, interleukin-23, follicle-stimulating hormone, angiopoietin-1 and eotaxin-1). In a prospective cohort of 354 patients undergoing peripheral and/or coronary angiography, performance of this diagnostic panel to detect $\geq 50 \%$ stenosis in at least one peripheral vessel was assessed in patients with $(n=94)$ and without DM $(n=260)$. The model had an area under the receiver operating characteristic curve (AUC) of 0.85 for obstructive PAD. At optimal cut-off, the model had $84 \%$ sensitivity, $75 \%$ specificity, positive predictive value (PPV) of $84 \%$ and negative predictive value (NPV) of $75 \%$ for detection of PAD among patients with DM, similar as in those without DM. In those with DM, partitioning the model into five levels resulted in a PPV of $95 \%$ and NPV of $100 \%$ in the highest and lowest levels, respectively. Abnormal scores were associated with a shorter time to revascularisation during 4.3 years of follow-up.

Conclusion A clinical/biomarker model can predict with high accuracy the presence of PAD among patients with DM.

Trial registration number NCT00842868.

\section{INTRODUCTION}

Diabetes mellitus (DM) is a global health problem; it is estimated, by 2030, approximately 366 million people worldwide will suffer from the disease. ${ }^{1}$ Patients with DM are at substantial risk for developing both microvascular and macrovascular complications. ${ }^{2}$ One notable macrovascular complication of DM is peripheral artery disease (PAD) which is prevalent in approximately $20 \%-30 \%$ of
Key questions

What is already known about this subject?

- The ankle-brachial index (ABI) is most commonly used to diagnose lower extremity peripheral artery disease (PAD); however, its diagnostic accuracy is limited in patients with stiff, calcified arteries which is common among patients with diabetes mellitus (DM).

- We recently developed a clinical/proteomic panel (HART PAD) using machine learning, capable of diagnosing obstructive PAD with high accuracy; however, the utility of this score in patients with DM is uncertain.

What does this study add?

- The HART PAD panel predicted with high accuracy the presence of PAD among patients with DM.

- Furthermore, the HART PAD panel was predictive of revascularisation among patients with DM.

How might this impact on clinical practice?

- The HART PAD panel offers an attractive alternative to $A B I$ for diagnosing PAD among patients with DM.

- The panel could act as a gatekeeper to imaging or invasive testing, thereby reducing costs, and exposures to intravenous contrast and/or ionising radiation by avoiding expensive imaging modalities when unwarranted.

- Furthermore, the panel could be used for prognostic purposes to guide more intensification of medical therapies.

patients. ${ }^{34}$ PAD is associated with a considerable increase in the risk of fatal and non-fatal cardiovascular and cerebrovascular events, and event rates are higher among patients with DM. ${ }^{6}$

Symptoms of PAD are variable, especially in patients with DM who may suffer from concomitant peripheral neuropathy, thus it is often undiagnosed until its advanced stages. As a result, patients with DM and PAD often receive suboptimal management that 
may prevent progression of disease. ${ }^{4}$ The ankle-brachial index (ABI) is the most common non-invasive diagnostic modality used to detect the presence of lower extremity PAD; however, its accuracy is reduced in patients with stiff, calcified arteries. Approximately $60 \%$ of patients with DM have calcified lower extremity peripheral arteries, and expectantly, ABI has correlated poorly with angiographic PAD in this population. ${ }^{7}$ Imaging modalities are also used to diagnose PAD but imaging is expensive, has variable availability and requires intravenous contrast and/or ionising radiation. For these reasons, we recently developed a clinical/proteomic panel (HART PAD) using machine learning, capable of diagnosing obstructive PAD with high accuracy. ${ }^{8}$ In this study, we compare the accuracy of this panel for the diagnosis of obstructive PAD in patients with and without DM a population at high risk for PAD that is particularly challenging to evaluate and manage.

\section{METHODS}

\section{Study population}

The Catheter Sampled Blood Archive in Cardiovascular Diseases study was a prospective, single-centre, observational cohort study that was undertaken at the Massachusetts General Hospital in Boston, Massachusetts, between 2008 and 2011. The investigators enrolled 1251 subjects undergoing coronary and peripheral angiography with or without intervention over the study period. ${ }^{9}$

For the purpose of this study, we included 354 patients who underwent peripheral angiography only $(n=140)$, peripheral and coronary angiography but without significant coronary artery disease (CAD) $(n=11)$ and those who underwent coronary angiography alone without significant CAD and no history of PAD $(n=203)$. The latter group were incorporated to increase cohort size and were assumed to have an absence of PAD, based on their medical history. The indications for peripheral angiography included claudication $(n=96)$, carotid artery stenosis with/without stroke $(n=11)$, hypertension $(n=21)$ and other PAD without claudication $(n=25)$. The peripheral angiograms included: lower extremity $(n=129)$, renal arteries $(n=59)$ and carotid/subclavian $(n=18)$. All study procedures were approved by the Partners HealthCare Institutional Review Board and carried out in accordance with the Declaration of Helsinki.

\section{Data acquisition}

After obtaining informed consent and at the time of the procedure, clinical and historical variables and reason for referral for angiography were recorded. Results of the peripheral +/-coronary angiography (based on visual estimation at the time of the procedure) were recorded. For the purposes of this analysis, $\geq 50 \%$ luminal obstruction in at least one peripheral vessel was considered obstructive PAD.

\section{HART PAD model}

The derivation of the HART PAD panel has been previously described. ${ }^{8}$ Briefly, using the same 354 patients included in this study, we used machine learning, a subset of artificial intelligence, to identify predictors of significant PAD. We examined 109 biomarkers and more than 50 clinical variables. Candidate panels of proteins and clinical features were generated via least angle regression. In this method, factors were included in the model one at a time, with their coefficients determined by their correlation with the outcome. This was repeated until all factors were included in the model, and the step at which the performance plateaued resulted in our initial panel of interest. With this panel of interest, predictive analyses were run on the training set using least absolute shrinkage and selection operator with logistic regression, predicting the outcome of obstructive PAD using only the variables in the panel of interest. If the contribution of the least performing variable was not statistically significant, then that variable was removed and the analysis rerun. This process was repeated until the predictive contribution of all variables in the model was statistically significant. This model development process was done via Monte Carlo cross-validation, using 400 iterations with an 80:20 (training:test) split. The final panel was used to create a final model with the entire sample, and this model was then evaluated to predict obstructive PAD.

After the machine learning model building process, the final panel consisted of one clinical variable (systemic hypertension) and six biomarkers: follicle-stimulating hormone, angiopoietin-1, kidney injury molecule-1 (KIM-1), midkine, interleukin-23 and eotaxin-1.

\section{Biomarker testing}

Using a centrally placed vascular access sheath, $15 \mathrm{~mL}$ of blood was taken immediately prior to angiography. Blood samples were then stored in a $4^{\circ} \mathrm{C}$ refrigerator until centrifuging was undertaken. After a single freezethaw cycle, $200 \mu \mathrm{L}$ of plasma was used for biomarker analysis on a Luminex 100/200 xMAP technology platform. The biomarkers were obtained from a commercially available kit, Myriad RBM MAP; this technology uses multiplexed, microsphere-based assays in a single reaction vessel whereby an assay-specific capture antibody on each microsphere binds to a protein of interest. Comparable to a flow cytometer, as each individual microsphere passes through a series of excitation beams, it is analysed for size, encoded fluorescence signature and the amount of fluorescence generated is proportionate to the protein concentration.

\section{Follow-up}

A review of patients' medical records from time of enrolment to the end of follow-up was undertaken. For identification of clinical endpoints, in addition to a review of medical records, telephone follow-up was performed with patients and/or their managing physicians. Study investigators adjudicating angiographic severity of PAD 
or events during follow-up were blinded to results of all biomarker testing.

\section{Statistical analysis}

Baseline characteristics between those with and without DM were compared; dichotomous variables were compared using two-sided Fisher's exact test, while continuous variables were compared using two-sided two-sample t-test. The biomarkers compared were tested with the Wilcoxon rank-sum test, as their concentrations were not normally distributed. A complete case analysis was performed; two patients were missing at least one of the concentration read-outs for the six proteins in the final panel, so they were excluded, leaving 352 samples available for analysis. For any biomarker result that was unmeasurable, we used a standard approach of imputing concentrations $50 \%$ below the limit of detection.

The HART PAD panel was originally developed to predict obstructive PAD using Monte Carlo cross-validation, and the final model was evaluated using in-sample validation. In this study, we used the final model trained to diagnose PAD in all patients, and evaluated it on all patients with DM. We generated a receiver operating characteristic curve and determined an optimal diagnostic cut-off using the optimal Youden's index. With this cut-off we calculated sensitivity, specificity, positive predictive value (PPV) and negative predictive value (NPV). The predictive score generated by the diagnostic model was rescaled to a range of $0-10$ to facilitate interpretation. The score was then partitioned into five different risk levels, corresponding to multiple levels of PAD risk. Time to revascularisation as a function of elevated PAD score was calculated from 7 days after index angiography over a mean of 4.3 years' follow-up period and displayed as a Kaplan-Meier survival curve. Lastly, after excluding patients with non-lower extremity PAD (eg, carotid and renal artery disease), we then assessed time to revascularisation or amputation in patients with lower extremity PAD as a function of the continuous HART PAD score (adjusting for age) over a mean of 4.3 years' follow-up period and displayed this as a Kaplan-Meier survival curve.

All statistics were performed by using $\mathrm{R}$ software, V.3.4 (R Foundation for Statistical Computing, Vienna, Austria). $P$ values are two sided, with a value $<0.05$ considered significant.

\section{RESULTS}

\section{Baseline characteristics}

Of the 354 patients included in this study, 132 had obstructive PAD (online supplementary table 1). Baseline characteristics of study subjects, dichotomised as a function of DM, are detailed in table 1. Patients with DM were older, more likely to be male and had a higher prevalence of hypertension, $\mathrm{CAD}$, prior myocardial infarction and chronic kidney disease. Notably, of the biomarkers measured, those with DM had higher concentrations of KIM-1 and midkine.

\section{HART PAD diagnostic performance}

With the data represented in the $0-10$ scale, the optimal cut-off for the panel was determined to be 5.607 using the optimal Youden's index, which identifies the optimal balance of sensitivity and specificity. In receiver operating characteristic testing, the model generated an in-sample AUC of 0.848 for patients with DM (figure 1); slightly higher than the performance for patients without DM (AUC 0.83).

At its optimal cut-off to diagnose $\mathrm{PAD}$, for patients with DM, we observed a sensitivity of $84 \%$, specificity of $75 \%$, NPV of $75 \%$ and PPV of $84 \%$.

\section{HART PAD five-level score}

Partitioning the score into five categories yielded a PPV of $95 \%$ and NPV of $100 \%$ in the highest and lowest scores, respectively, for patients with DM (table 2). We found a higher prevalence of obstructive PAD in those with higher scores and lower prevalence among those with lower scores (figure 2). When the score was divided into five categories of predicted risk, an increasing score correlated with an increasing degree of mean PAD stenosis in both patients with and without DM (figure 3).

\section{Performance in various vascular territories}

The score had a similar in-sample performance in each individual vascular territory using the optimal cut-off among patients with DM. In the diagnosis of obstructive lower extremity artery disease, the score had a sensitivity of $83 \%$, specificity of $62 \%$, PPV of $70 \%$ and NPV of $78 \%$. For diagnosing obstructive carotid and renal artery stenosis, the score appeared most useful for its NPV. The score had a sensitivity of $80 \%$, specificity of $58 \%$, PPV of $10 \%$ and NPV of $98 \%$ for obstructive carotid arterial disease. While for the renal arteries, the corresponding sensitivity was $88 \%$, specificity $59 \%$, PPV $17 \%$ and NPV $98 \%$.

\section{Predicting the need for revascularisation}

In adjusted Cox proportional hazards models, from 7 days after index angiography to the end of follow-up, those patients with DM who had a dichotomously elevated score had higher risk for revascularisation, compared with patients with DM who had a lower PAD score (HR: 2.88; $95 \%$ CI 1.16 to $7.19, \mathrm{p}=0.02$ ); those with higher scores also had shorter time to first revascularisation event (figure 4). After excluding patients with obstructive PAD in non-lower extremity territories (figure 5), the score predicted time to revascularisation or amputation as a continuous score among patients with DM and lower extremity PAD (HR: 1.3; 95\% CI 1.02 to $1.66, \mathrm{p}=0.04$ ).

\section{DISCUSSION}

Though prevalent in patients with DM, PAD is a challenge to identify and manage; tools typically used for 
Table 1 Baseline characteristics of patients with and without diabetes mellitus

\begin{tabular}{|c|c|c|c|}
\hline Characteristics & $\begin{array}{l}\text { Subjects with DM } \\
(\mathrm{n}=94)\end{array}$ & $\begin{array}{l}\text { Subjects without DM } \\
(n=260)\end{array}$ & $P$ value \\
\hline \multicolumn{4}{|l|}{ Demographics } \\
\hline Age (years) & $68 \pm 11.3$ & $63 \pm 8.4$ & 0.002 \\
\hline Male sex & $74.5 \%$ & $56.9 \%$ & 0.003 \\
\hline Caucasian & $92.6 \%$ & $92.3 \%$ & 1.00 \\
\hline \multicolumn{4}{|l|}{ Medical history } \\
\hline Smoker & $15.2 \%$ & $15.5 \%$ & 1.00 \\
\hline Atrial fibrillation/flutter & $25.5 \%$ & $21.2 \%$ & 0.39 \\
\hline Hypertension & 91.5 & $69.6 \%$ & $<0.001$ \\
\hline Coronary artery disease & $58.5 \%$ & $28.46 \%$ & $<0.001$ \\
\hline Prior Ml & $14.9 \%$ & $13.5 \%$ & $<0.001$ \\
\hline Heart failure & $30.9 \%$ & $19.6 \%$ & 0.24 \\
\hline COPD & $21.2 \%$ & $20.2 \%$ & 0.89 \\
\hline CVA/TIA & $22.3 \%$ & $8.5 \%$ & $<0.001$ \\
\hline CKD & $27.7 \%$ & $4.2 \%$ & $<0.001$ \\
\hline Prior CABG & $29.8 \%$ & $9.2 \%$ & $<0.001$ \\
\hline Prior percutaneous coronary intervention & $40.4 \%$ & $22.3 \%$ & 0.001 \\
\hline \multicolumn{4}{|l|}{ Medications } \\
\hline ACE-I/ARB & $70.2 \%$ & $47.3 \%$ & $<0.001$ \\
\hline Beta blocker & $74.5 \%$ & $56.8 \%$ & 0.003 \\
\hline Aldosterone antagonist & $4.3 \%$ & $3.5 \%$ & 0.75 \\
\hline Loop diuretics & $31.9 \%$ & $16.6 \%$ & 0.003 \\
\hline Nitrates & $17.0 \%$ & $10.8 \%$ & 0.14 \\
\hline Statin & $81.9 \%$ & $61.4 \%$ & $<0.001$ \\
\hline Aspirin & $78.7 \%$ & $68.0 \%$ & 0.06 \\
\hline Warfarin & $21.3 \%$ & $18.6 \%$ & 0.65 \\
\hline Clopidogrel & $27.7 \%$ & $14.7 \%$ & 0.008 \\
\hline \multicolumn{4}{|l|}{ Biomarkers } \\
\hline Angiopoietin-1 (ng/mL), median (25th-75th percentiles) & $6.4(4.3,8.58)$ & $6.8(5,11)$ & 0.05 \\
\hline Eotaxin-1 (pg/mL), median (25th-75th percentiles) & $108(42.5,150.5)$ & $97(42.5,144)$ & 0.10 \\
\hline $\begin{array}{l}\text { Follicle-stimulating hormone }(\mathrm{mlU} / \mathrm{mL}) \text {, median }(25 \text { th- } \\
\text { 75th percentiles) }\end{array}$ & $8.7(4.55,29.25)$ & $9.7(4.15,41.5)$ & 0.38 \\
\hline Interleukin-23 (ng/mL), median (25th-75th percentiles) & $2.8(2.23,3.38)$ & $2.5(2,3.2)$ & 0.06 \\
\hline $\begin{array}{l}\text { Kidney injury molecule-1 (ng/mL), median (25th-75th } \\
\text { percentiles) }\end{array}$ & $0.07(0.04,0.12)$ & $0.01(0.01,0.05)$ & $<0.001$ \\
\hline Midkine (ng/mL), median (25th-75th percentiles) & $18(13.25,26)$ & $13(9.9,19)$ & $<0.001$ \\
\hline
\end{tabular}

All continuous variables are displayed as mean $\pm S D$, unless otherwise specified.

ACE-I/ARB, ACE inhibitor/angiotensin receptor blocker; CABG, coronary artery bypass graft; CKD, chronic kidney disease; COPD, chronic obstructive pulmonary disease; CVA/TIA, cerebrovascular accident/transient ischaemic attack;DM, diabetes mellitus; MI, myocardial infarction.

diagnosis of PAD are often less accurate in those with DM. Accordingly, we wished to verify the performance of a biomarker-leveraged scoring system derived using machine learning recently found to predict angiographically significant PAD. ${ }^{8}$ We demonstrate excellent performance of the model in patients with DM. When analysed as a five-level score, the highest and lowest scores yielded a PPV of $95 \%$ and NPV of $100 \%$, respectively, for diagnosing obstructive PAD among patients with DM.

As discussed, the biomarkers used in this model all have credible biological links to atherosclerotic PAD. ${ }^{10-19}$ We believe the HART PAD panel could be useful in those with DM. As $60 \%$ of patients with DM have calcified lower extremity peripheral arteries, ABI, the most commonly 


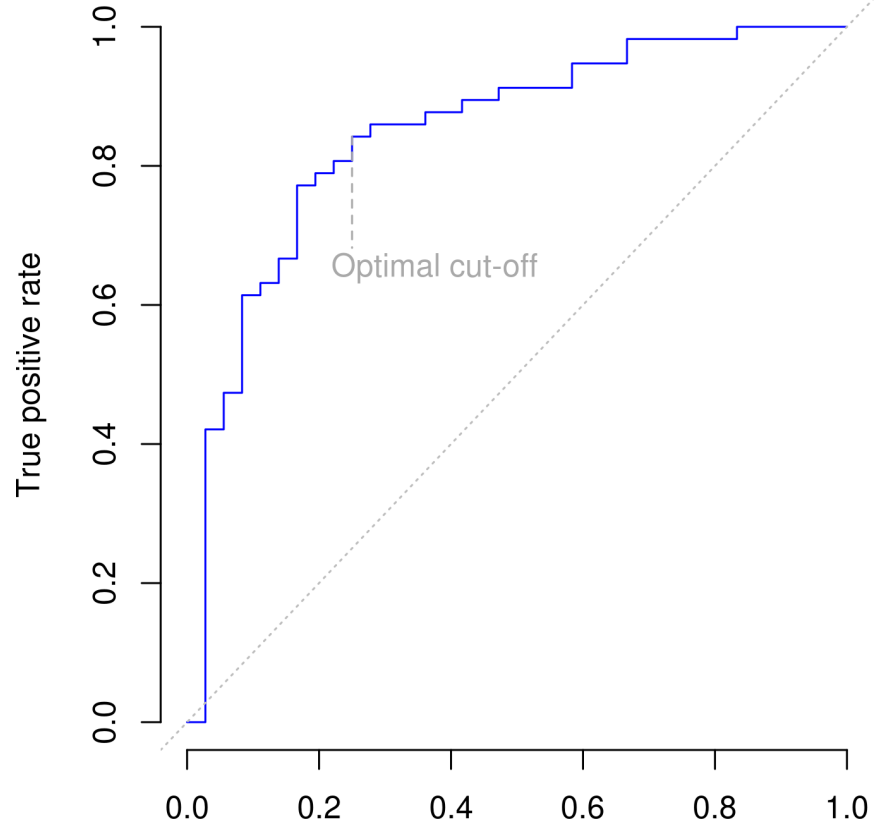

False positive rate

$(\mathrm{AUC}=0.848)$

Figure 1 Receiver operating characteristic curve for the HART PAD score to predict obstructive peripheral arterial disease in patients with diabetes mellitus. The score had a very robust area under the receiver operating characteristic curve (AUC).

used non-invasive diagnostic tool, has correlated poorly with angiographic PAD in this population. ${ }^{7}$ The HART PAD panel offers an attractive alternative to ABI with high NPV and PPV in patients with DM. Given a range of score values that provides both strong PPV and NPV, the panel could potentially avoid the need for imaging or invasive testing, thereby reducing costs, and exposures to intravenous contrast and/or ionising radiation. Of note, the model performed similarly well in other vascular regions (renal and carotid arteries), in particular for its NPV. Undoubtedly, the panel should serve as an adjunct to a thorough history and physical examination.

Furthermore, we demonstrate that the HART PAD panel has prognostic utility, with a shorter time to revascularisation in those with an elevated score. Consequently, the scoring system may also be used to predict patients at risk for vascular complications, which could then be used to guide therapeutic intervention. Aspirin and

\begin{tabular}{lll}
\hline Table 2 & Predictive performance as a five-level score \\
\hline Score & $\begin{array}{l}\text { Positive predictive } \\
\text { value }\end{array}$ & $\begin{array}{l}\text { Negative predictive } \\
\text { value }\end{array}$ \\
\hline 5 & 0.95 & - \\
4 & 0.66 & - \\
3 & 0.31 & 0.69 \\
2 & - & 0.86 \\
1 & - & 1.00 \\
\hline
\end{tabular}

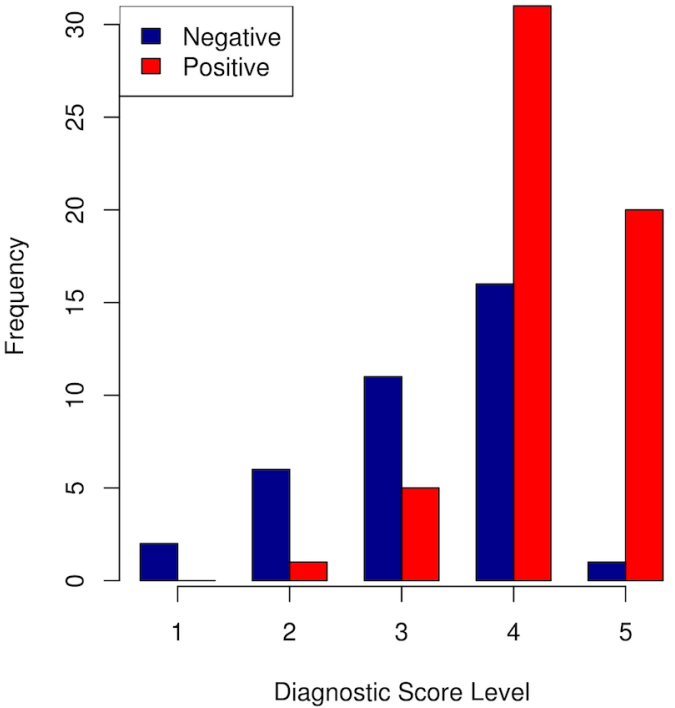

Figure 2 Distribution of score among patients with diabetes (positive) and without diabetes (negative) in a histogram.

statins are already standard therapies in PAD, but other medications such as novel oral anticoagulants (NOAC) and proprotein convertase subtilisin/kexin type 9 (PCSK9) inhibitors have recently demonstrated promise in this population. ${ }^{2021}$ Cost (PCSK9 inhibitors) and side effect profile (NOACs) may limit their utility and, thus, appropriate risk stratification will be important to guide therapy.

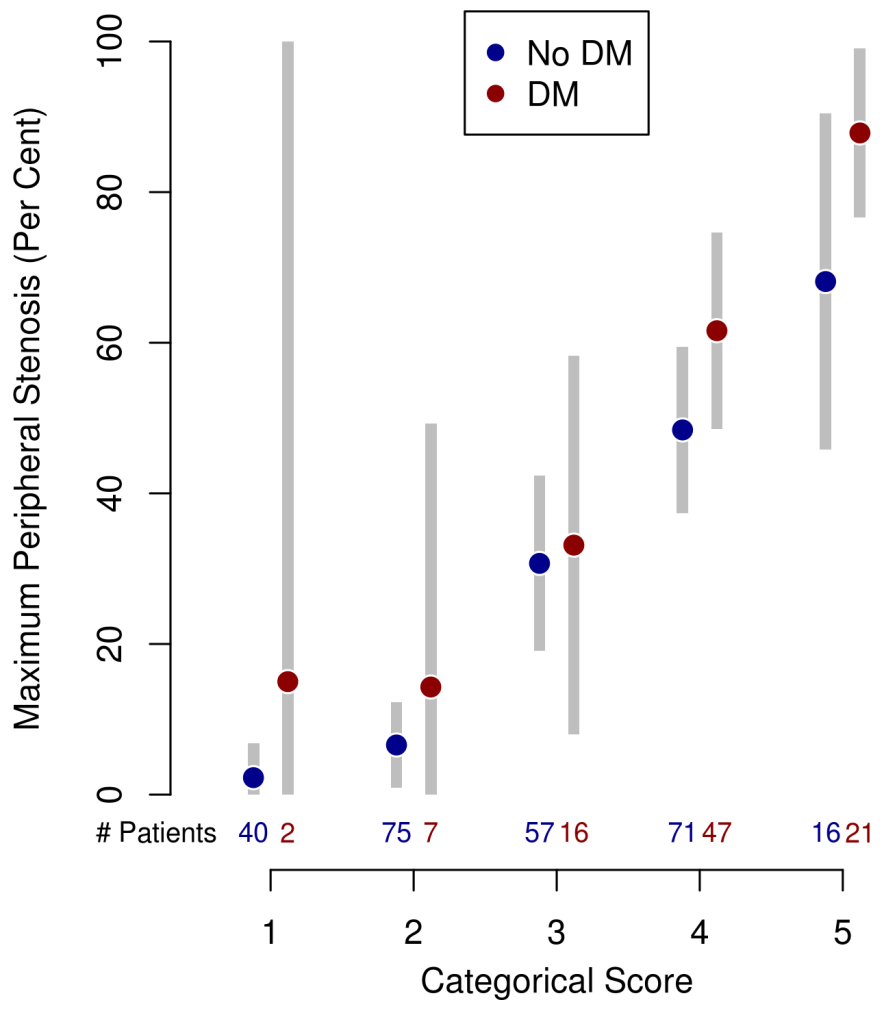

Figure 3 Correlation between peripheral artery disease (PAD) score and mean degree of arterial stenosis in patients with and without diabetes mellitus (DM). 


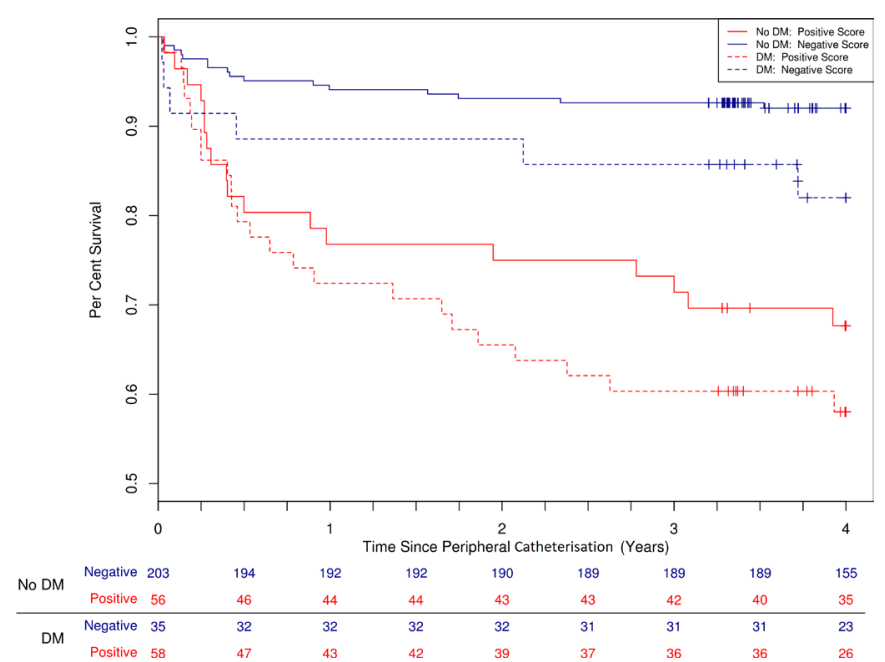

Figure 4 Kaplan-Meier survival curves depicting time to revascularisation as a function of peripheral artery disease (PAD) score. Patients in the positive group had a score greater than or equal to the optimal cut-off for the score, which was determined to be 5.607 using the optimal Youden's index (with the model's output rescaled to the range of $0-10$ ). Patients in the negative group had a score below 5.607. DM, diabetes mellitus.

Lastly, the HART PAD panel may play a role in clinical trials to enrich for PAD-related events or to identify patients at risk for adverse effects of drug therapies. For example, although the sodium-glucose co-transporter-2 inhibitor canagliflozin significantly reduced the risk of cardiovascular events by $14 \%$, it doubled the risk of amputation in patients with type $2 \mathrm{DM}$. Our panel could be useful in predicting underlying PAD or the need for

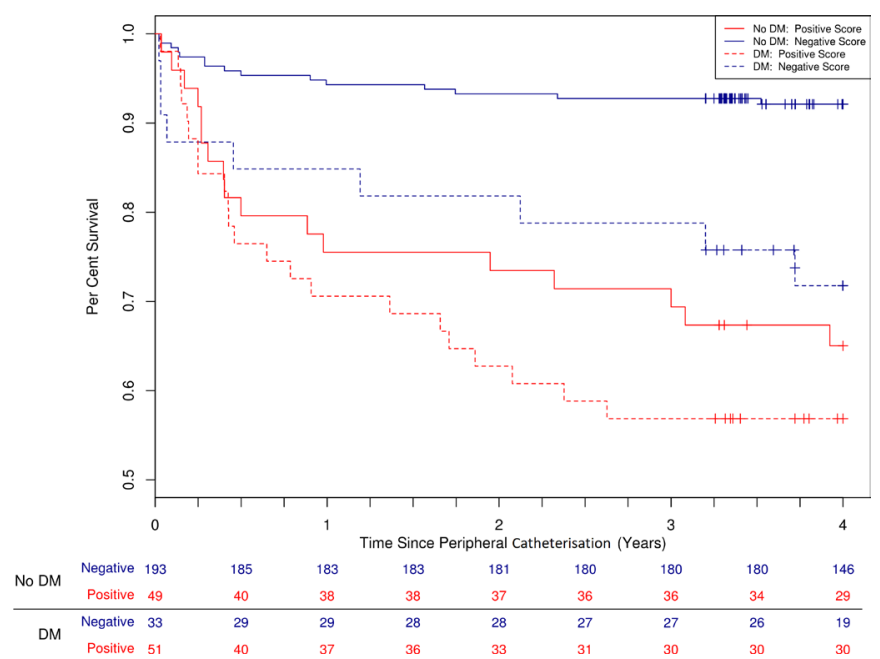

Figure 5 Kaplan-Meier survival curves depicting time to revascularisation as a function of continuous HART PAD score in patients with lower extremity peripheral artery disease (PAD). Patients in the positive group had a score greater than or equal to the optimal cut-off for the score, which was determined to be 5.607 using the optimal Youden's index (with the model's output rescaled to the range of $0-10$ ). Patients in the negative group had a score below 5.607. DM, diabetes mellitus. revascularisation in patients who are being considered for these agents. ${ }^{22}$

Despite being novel, our study has limitations. Notably, the number of patients from which we derived our findings was relatively small and included patients who only underwent coronary angiography as negative controls; though such patients were low risk for PAD, unexpected disease might have been present. As such, the results of our study should serve as preliminary evidence that requires confirmation in larger and ad hoc cohorts. The study participants were predominantly Caucasian which limits to the external validity of our panel to African-American patients. Patients with critical limb ischaemia were not included in our study and the utility of the panel in this cohort is uncertain. The diagnostic model was not compared with other non-invasive modalities such as ABI or ultrasonography; a comparison which will necessitate investigation in future studies. Our results need further validation and should not be extrapolated to the general population; the patients in our study were referred with clinical suspicion for significant PAD. Lastly, using the clinical/biomarker panel alone will not be sufficient to differentiate the exact territory of PAD and thus clinical correlation with history and physical examination will be an important component of assessment.

\section{CONCLUSION}

We describe a clinical/biomarker panel with high accuracy for predicting the presence of PAD in patients with DM.

Contributors All authors played a role in the drafting and editing of the manuscript.

Funding $\mathrm{NI}$ is supported by the Dennis and Marilyn Barry Fellowship in Cardiology Research. JLJ is supported in part by the Hutter Family Professorship in Cardiology. HKG is supported in part by the Ruth and James Clark Fund for Cardiac Research Innovation. This work was supported by a grant from Prevencio.

Competing interests JLJ has received grant support from Abbott, Cleveland Heart Labs, Singulex and Prevencio; has received consulting income from Roche Diagnostics, Critical Diagnostics and Novartis; and has participated in clinical endpoint committees/data or safety monitoring boards for Novartis, Amgen, GE, Janssen, Pfizer and Boehringer Ingelheim. CM is a consultant to Prevencio. HKG has received grant support from Roche and Portola; consulting income from Roche Diagnostics, American Regent, Amgen, Boston Heart Diagnostics and Critical Diagnostics; research payments for clinical endpoint committees for EchoSense. JMG has received consulting income from Siemens, Applied Clinical Intelligence, Bayer and Merck, Boehringer Ingelheim and AbbVie. NI has received speaker fees from Novartis. RRJvK has received grant support from Novartis. RR and GB are employees of Prevencio.

Patient consent for publication Obtained.

Ethics approval Partners HealthCare Institutional Review Board (Study ID No: 2008P001076).

Provenance and peer review Not commissioned; externally peer reviewed.

Data availability statement All data relevant to the study are included in the article or uploaded as supplementary information.

Open access This is an open access article distributed in accordance with the Creative Commons Attribution Non Commercial (CC BY-NC 4.0) license, which permits others to distribute, remix, adapt, build upon this work non-commercially, and license their derivative works on different terms, provided the original work is properly cited, appropriate credit is given, any changes made indicated, and the use is non-commercial. See: http://creativecommons.org/licenses/by-nc/4.0/. 


\section{REFERENCES}

1. Wild S, Roglic G, Green A, et al. Global prevalence of diabetes: estimates for the year 2000 and projections for 2030. Diabetes Care 2004;27:1047-53.

2. Beckman JA, Creager MA, Libby P. Diabetes and atherosclerosis: epidemiology, pathophysiology, and management. JAMA 2002;287:2570-81.

3. Elhadd TA, Robb R, Jung RT, et al. Pilot study of prevalence of asymptomatic peripheral arterial occlusive disease in patients with diabetes attending a hospital clinic. Pract. Diab. Int. 1999;16:163-6.

4. Hirsch AT, Criqui MH, Treat-Jacobson D, et al. Peripheral arterial disease detection, awareness, and treatment in primary care. JAMA 2001;286:1317-24.

5. Weitz Jl, Byrne J, Clagett GP, et al. Diagnosis and treatment of chronic arterial insufficiency of the lower extremities: a critical review. Circulation 1996;94:3026-49.

6. American Diabetes Association. Peripheral arterial disease in people with diabetes. Diabetes Care 2003;26:3333-41.

7. Aerden D, Massaad D, von Kemp K, et al. The ankle--brachial index and the diabetic foot: a troublesome marriage. Ann Vasc Surg 2011;25:770-7.

8. McCarthy CP, Ibrahim NE, van Kimmenade RRJ, et al. A clinical and proteomics approach to predict the presence of obstructive peripheral arterial disease: from the catheter sampled blood Archive in cardiovascular diseases (Casablanca) study. Clin Cardiol 2018;41:903-9.

9. Gaggin HK, Bhardwaj A, Belcher AM, et al. Design, methods, baseline characteristics and interim results of the catheter sampled blood Archive in cardiovascular diseases (Casablanca) study. IJC Metab Endocr 2014:5:11-18.

10. Abbas A, Gregersen I, Holm S, et al. Interleukin 23 levels are increased in carotid atherosclerosis: possible role for the interleukin 23/interleukin 17 axis. Stroke 2015;46:793-9.

11. Salaru DL, Albert C, Königsmark U, et al. Serum levels for midkine, a heparin-binding growth factor, inversely correlate with angiotensin and endothelin receptor autoantibody titers in patients with macroangiopathy. Int Angiol 2014;33:372-8.
12. Salaru DL, Arsenescu-Georgescu C, Chatzikyrkou C, et al. Midkine, a heparin-binding growth factor, and its roles in atherogenesis and inflammatory kidney diseases. Nephrol Dial Transplant 2016;31:1781-7.

13. Haley KJ, Lilly CM, Yang JH, et al. Overexpression of eotaxin and the CCR3 receptor in human atherosclerosis: using genomic technology to identify a potential novel pathway of vascular inflammation. Circulation 2000;102:2185-9.

14. Emanuele $E$, Falcone $C, D^{\prime}$ Angelo $A$, et al. Association of plasma eotaxin levels with the presence and extent of angiographic coronary artery disease. Atherosclerosis 2006;186:140-5.

15. David A, Saitta S, De Caridi G, et al. Interleukin-23 serum levels in patients affected by peripheral arterial disease. Clin Biochem 2012;45:275-8.

16. Ibrahim NE, Januzzi JL, Magaret CA, et al. A clinical and biomarker scoring system to predict the presence of obstructive coronary artery disease. J Am Coll Cardiol 2017;69:1147-56.

17. Bertone-Johnson ER, Virtanen JK, Nurmi T, et al. Folliclestimulating hormone levels and subclinical atherosclerosis in older postmenopausal women. Am J Epidemiol 2018;187:16-26.

18. Wang $\mathrm{N}$, Shao $\mathrm{H}$, Chen $\mathrm{Y}$, et al. Follicle-stimulating hormone, its association with cardiometabolic risk factors, and 10-year risk of cardiovascular disease in postmenopausal women. J Am Heart Assoc 2017;6:e005918.

19. Lim HS, Blann AD, Chong AY, et al. Plasma vascular endothelial growth factor, angiopoietin-1, and angiopoietin-2 in diabetes: implications for cardiovascular risk and effects of multifactorial intervention. Diabetes Care 2004;27:2918-24.

20. Eikelboom JW, Connolly SJ, Bosch J, et al. Rivaroxaban with or without aspirin in stable cardiovascular disease. $N$ Engl J Med 2017;377:1319-30.

21. Bonaca MP, Nault P, Giugliano RP, et al. Low-density lipoprotein cholesterol lowering with evolocumab and outcomes in patients with peripheral artery disease: insights from the Fourier trial (Further cardiovascular outcomes research with PCSK9 inhibition in subjects with elevated risk). Circulation 2018;137:338-50.

22. Neal B, Perkovic V, Mahaffey KW, et al. Canagliflozin and cardiovascular and renal events in type 2 diabetes. $N$ Engl J Med 2017;377:644-57. 